\title{
ASPECTOS GERAIS DA SEXUALIDADE DOS PORTADORES DE TUBERCULOSE PULMONAR ATENDIDOS EM UNIDADES BÁSICAS DE SAÚDE DE RIBEIRÃO PRETO-SP*
}

\author{
Érika do Carmo Bertazone** \\ Elucir Gir***
}

\begin{abstract}
BERTAZONE, E.do C.; GIR, E. Aspectos gerais da sexualidade dos portadores de tuberculose pulmonar atendidos em unidades básicas de saúde de Ribeirão Preto-SP. Rev.latino-am.enfermagem, Ribeirão Preto, v. 8, n. 1, p. 115-122, janeiro 2000.
\end{abstract}

Este estudo teve como objetivos identificar e analisar o conhecimento que os portadores de tuberculose pulmonar têm acerca da doença e as experiências vivenciadas por estes, enfatizando-se as modificações ocorridas no exercício da sexualidade, as reações dos familiares/amigos e os sentimentos emergentes a partir destas experiências. Foram entrevistados cinqüenta pacientes, sexualmente ativos, portadores de tuberculose pulmonar. Os resultados evidenciaram que 15 (30,0\%) dos portadores de tuberculose pulmonar não têm conhecimento a respeito da doença e 32 (64,0\%) não sabem como prevenir a mesma. Dos 50 entrevistados, 29 $(58,0 \%)$ perceberam reações negativas, onde o medo do próprio portador de transmitir a infecção/doença, bem como o medo da parceria sexual em adquirir a infecção/doença, pode causar alterações na sua vida social, afetiva elou sexual, resultando na diminuição da freqüencia das relações sexuais, afetando assim a sua relação.

UNITERMOS: tuberculose pulmonar, emoções, enfermagem

\section{INTRODUÇÃO}

A Tuberculose Pulmonar no Brasil continua sendo um sério problema de Saúde Pública. Os avanços no seu conhecimento e os meios de diagnóstico disponíveis para o seu controle não têm sido suficientes para diminuir ou estabilizar a sua morbidade e mortalidade.

$\mathrm{Na}$ verdade o que ocorre é que há grande dificuldade para se controlar a doença devido à pobreza, à epidemia de AIDS e a deterioração dos serviços de saúde, pois os mesmos são fatores que contribuem expressivamente para o recrudescimento desta doença.

Mas, o que não se esperava, é que a tuberculose pulmonar ressurgisse às custas da epidemia de AIDS, tornando-se assim mais resistente e contribuindo consideravelmente para o aumento do número de casos. No Brasil, desde a década de 80 , vem sendo notificados de 80 a 90.000 casos novos de tuberculose, além de aproximadamente $10 \%$ a mais de casos de retratamento (HIJJAR, 1997).
No ano de 1996, no Estado de São Paulo, de acordo com dados do BRASIL.SUS (1997), foram notificados, até o $1^{\circ}$ trimestre de $1997,14.375$ casos novos de tuberculose pulmonar. Já no ano de 1997, na cidade de Ribeirão Preto, foram notificados 357 casos.

Tendo em vista a progressão desta doença, é imprescindível que atenção especial seja dada à sua disseminação, pois a mesma demonstra uma grande habilidade em ressurgir, mesmo depois de um longo período de estabilidade.

Face a estas variáveis, a tuberculose pulmonar vem sendo caracterizada como uma doença crônica ressurgente, levando os seus portadores a experienciarem preconceito ou até mesmo isolamento social.

MATA \& JOSE (1985), em uma pesquisa realizada em Honduras, colocam que um indivíduo envolvido com Tuberculose Pulmonar tem uma imagem negativa da doença e conseqüentemente experiência rejeição ou algum tipo de sentimento ligado a ela.

Como observamos, a Tuberculose Pulmonar pode afetar a vida de um indivíduo, refletindo

\footnotetext{
* Resumo da Tese de Mestrado da autora: "O exercício da sexualidade de portadores de tuberculose pulmonar atendidos em unidades básicas de saúde de Ribeirão Preto-SP”, apresentada à Escola de Enfermagem de Ribeirão Preto da Universidade de São Paulo. Ribeirão Preto, 1998

** Enfermeira. Mestre em Enfermagem junto à Escola de Enfermagem de Ribeirão Preto da Universidade de São Paulo

*** Enfermeira. Professor Associado junto ao Departamento de Enfermagem Geral e Especializada da Escola de Enfermagem de Ribeirão Preto da Universidade de São Paulo
} 
conseqüentemente, nas relações que o(a) portador(a) tem com outras pessoas, inclusive no exercício de sua sexualidade.

A experiência profissional nos mostra que portadores de tuberculose pulmonar sofrem com a aquisição desta doença, não só pelas manifestações clínicas, mas também pela possibilidade de vivenciar preconceito e rejeição, afetando conseqüentemente as relações sociais em geral. Dentre tais relações destacase a relação com a parceria sexual, tornando-a susceptível a alterações causadas por fatores de ordem sócioemocional.

Partindo do pressuposto de que podem ocorrer modificações no comportamento e conseqüentemente nas relações dos portadores de tuberculose pulmonar, após a manifestação da doença, este estudo teve como objetivos gerais:

- Identificar e analisar o conhecimento que os portadores de tuberculose pulmonar, atendidos em Unidades Básicas de Saúde do município de Ribeirão Preto tem acerca da doença, bem como as experiências vivenciadas por estes no decorrer da mesma.

- Caracterizar e comparar os resultados dos depoimentos de portadores de tuberculose pulmonar, atendidos em Unidades Básicas de Saúde do município de Ribeirão Preto, acerca do exercício de sua sexualidade, considerando-se dois períodos: antes e após a instalação da doença.

Como objetivos específicos o estudo visou:

- Analisar o conhecimento que os portadores de tuberculose pulmonar têm acerca da doença, no que diz respeito à nomenclatura da doença, formas de transmissão, medidas de prevenção, sinais e sintomas, gravidade, fatores de risco e cura.

- Caracterizar as experiências vivenciadas relativas ao convívio familiar, às reações dos familiares/amigos e os sentimentos emergentes no decorrer da doença.

- Analisar a ocorrência de mudanças no exercício da sexualidade dos portadores de tuberculose pulmonar, após a manifestação da doença.

\section{METODOLOGIA}

Neste estudo exploratório descritivo, preocupouse em identificar e analisar aspectos relacionados ao conhecimento que os portadores de tuberculose pulmonar têm acerca da doença, a ocorrência de mudanças no exercício de sua sexualidade e caracterizar as experiências vivenciadas por eles relativas ao convívio familiar, reações dos familiares/amigos e sentimentos emergentes no decorrer da doença.
O presente estudo foi realizado junto aos indivíduos portadores de Tuberculose Pulmonar, diagnosticada há pelo menos 3 meses, que estavam em tratamento e/ou acompanhamento em três Unidades Básicas de Saúde do município de Ribeirão Preto - São Paulo, no período de janeiro a agosto de 1997.

A população foi constituída de indivíduos do sexo masculino e feminino, com diagnóstico médico de Tuberculose Pulmonar, definido há pelo menos 3 meses, residentes em Ribeirão Preto e que estavam em tratamento e/ou acompanhamento clínico nas Unidades A, B e C.

Da amostra, fizeram parte indivíduos do sexo masculino e feminino, na faixa etária de 15 a 64 anos, portadores de Tuberculose Pulmonar e sabedores do seu diagnóstico há pelo menos 3 meses, que eram, ou já haviam sido ativos sexualmente, tendo parceria fixa ou não, independente do estado marital.

Foram excluídos os indivíduos que apresentavam tuberculose extra-pulmonar e/ou tuberculose pulmonar associada à Síndrome de Imunodeficiência Adquirida (AIDS).

Os dados foram coletados pela própria pesquisadora, através de entrevista semi-diretiva gravada, norteada por um questionário composto por questões abertas e fechadas, após a aquiescência dos entrevistados, mediante a assinatura de um termo de consentimento.

Os dados foram analisados qualiquantitativamente e submetidos a cálculos de porcentagem simples. Para analisar as questões referentes ao modo de transmissão e prevenção, foram criados enunciados a partir das respostas.

\section{RESULTADOS E DISCUSSÃO}

Dos 50 pacientes portadores de tuberculose pulmonar, sujeitos desta investigação, atendidos nas 3 unidades básicas de saúde, $40(80 \%)$ pacientes são do sexo masculino e $10(20 \%)$ do sexo feminino.

Quanto à cor, 27 (54,0\%) são brancos, 7 (14,0\%) pretos, $15(30,0 \%)$ pardos e $1(2,0 \%)$ é amarelo.

No que se refere à idade dos pacientes, observamos a predominância de $8(16,0 \%)$ pacientes entre 20 e 24 anos e 11 meses; 9 (18,0\%) entre 40 e 44 anos e 11 meses e $7(14,0 \%)$ pacientes entre 50 e 54 anos e 11 meses. Os limites inferior e superior se referem a um paciente com 17 anos de idade e um com 64 anos, respectivamente.

No que concerne ao estado civil, $13(26,0 \%)$ pacientes são solteiros, $18(36,0 \%)$ casados, $4(8,0 \%)$ descasados****, $2(4,0 \%)$ viúvos e $13(26,0 \%)$ são amasiados, valendo ressaltar que $42(84,0 \%)$ pacientes

**** Entende-se por descasados os indivíduos que viviam com suas esposas ou amasiadas e separaram-se legal ou ilegalmente das mesmas 
referiram ter parceria sexual no momento da entrevista e $8(16,0 \%)$ referiram não ter.

Com respeito à escolaridade, $1(2,0 \%)$ paciente referiu ter concluído o primeiro grau, $37(74,0 \%)$ não o fizeram; 4 (8,0\%) concluíram o segundo grau e $2(4,0 \%)$ mencionaram apresentar escolaridade secundária incompleta. Quanto ao terceiro grau, $1(2,0 \%)$ paciente o terminou e $4(8,0 \%)$ apresentaram terceiro grau incompleto. Os $4(8,0 \%)$ restantes referiram ser analfabetos, mas capazes de assinar seus nomes.

Em relação à identidade sexual, destacamos que $47(94 \%)$ referiram ser heterossexuais, 2 (4,0\%) bissexuais e $1(2,0 \%)$ homossexual.

Concernente à profissão, observamos que há predominância entre as profissões que se relacionam à serventia, segurança e estética $24(48,0 \%)$ e comércio 8 $(16,0 \%)$. Ressaltamos que dos $24(48,0 \%)$ pacientes que pertencem ao agrupamento serventia, segurança e estética, encontramos $12(24,0 \%)$ pedreiros, $4(8,0 \%)$ domésticas, $2(4,0 \%)$ do lar, $2(4,0 \%)$ vigias, $1(2,0 \%)$ marceneiro, $1(2,0 \%)$ instalador de telefone, $1(2,0 \%)$ camareira e $1(2,0 \%)$ é armador.

Podemos relacionar a susceptibilidade à doença à profissão destes indivíduos, pois determinadas profissões como pedreiros e serventes, requerem um grande esforço físico por parte dos mesmos, aliado à má alimentação e a vida desregrada.

Muitos indivíduos trabalham em locais fechados, com várias pessoas, desconhecendo os sinais e sintomas da tuberculose pulmonar. Se houvesse um conhecimento e maior atenção direcionados a tal doença, seria maior a possibilidade de se encaminhar tais indivíduos a um tratamento precoce.

Concernente ao conhecimento que os pacientes têm sobre a doença propriamente dita, 41 pacientes, ou seja, $82,0 \%$ referiram conhecer a tuberculose pulmonar por este próprio nome, enquanto que $9(18,0 \%)$ pacientes atribuem outras denominações a ela, tais como "doença do pulmão", pneumonia, tuberculose galopante, "problema do coração" e enfisema pulmonar. É curioso notar que $2(4,0 \%)$ pacientes afirmaram que "a tuberculose é a AIDS".

Talvez a relação feita pelos indivíduos, entre a tuberculose e a AIDS, esteja relacionada ao teor das mensagens veiculadas nos meios de comunicação. Ao mesmo tempo, tal relação possa ter sua justificativa, no fato de a tuberculose pulmonar complicar, com freqüência, a infecção pelo HIV (SCHAFFNER, 1996).

No que se refere ao modo de transmissão, encontramos que $21(42,0 \%)$ dos pacientes acreditam que a transmissão da mesma ocorra pelas vias aéreas através da respiração, tosse, espirro, saliva, ar e pulmões; 8 $(16,0 \%)$ acreditam que ela seja transmitida através do contato com material contaminado, como objetos, alimentos, beijo ou "relação sexual não normal" (sexo oral); $2(4,0 \%)$ referiram não ter tido orientação médica; $2(4,0 \%)$ mencionaram crenças e 15 pacientes $(30 \%)$ referiram não saber e não ter idéia a respeito do modo de transmissão da tuberculose pulmonar.

Os dados evidenciaram que grande parte dos pacientes têm uma noção vaga no que concerne ao modo de transmissão da referida patologia.

Acreditamos que comunicações informais, que as pessoas têm no seu convívio diário, contribuem para que as mesmas absorvam principalmente conhecimentos de senso comum, que podem ser entendidos de maneira incorreta.

Ressaltamos ainda, que no processo de comunicação, mesmo quando as informações são emitidas por um profissional da saúde, podem ocorrer ruídos, o que pode levar a interpretações indevidas.

Com respeito às medidas de prevenção, 26 $(52,0 \%)$ pacientes afirmaram ter sido vacinados, $4(8,0 \%)$ referiram não ter recebido a vacina e os $20(40,0 \%)$ restantes não souberam informar se haviam sido vacinados ou não.

Quando questionados a respeito do conhecimento que têm sobre a vacina, $36(72,0 \%)$ referiram não saber nada a respeito da mesma.

Percebemos então, que as crenças, como por exemplo as mencionadas pelos pacientes, de que a doença se transmite sexualmente, em relações sexuais "não normais", ou seja sexo oral; se o indivíduo ingerir água quente ou tomar banho quente e após "pegar friagem" ele adquire a doença e de que a tuberculose pulmonar é uma doença hereditária, influem fortemente nos conceitos e nas atitudes que os pacientes têm, levando-os a desacreditar nas verdadeiras causas de transmissão e a desconhecer o real significado das coisas.

É interessante notar que a falta de interesse ou indiferença pela própria doença é visível, mesmo em indivíduos que se encontram em tratamento. A grande maioria chega ao ponto de não saber se prevenir contra recidivas da doença, a qual está comprometendo o seu organismo.

Quando questionados quanto à gravidade da doença, $37(74,0 \%)$ pacientes acreditam que a tuberculose pulmonar seja uma doença grave; $12(24,0 \%)$ não acreditam na sua gravidade e $1(2,0 \%)$ não sabe opinar.

Ainda discutindo o aspecto da prevenção, detectamos entre os entrevistados que, $32(64,0 \%)$ não sabem como prevenir a doença e $18(36,0 \%)$ restantes referiram conhecer algumas medidas preventivas.

No que concerne aos sentimentos e emoções apresentados pelos respondentes, observamos que o que realmente prevalece são os sentimentos negativos. $\mathrm{O}$ medo foi mencionado por $29(58,0 \%)$ pacientes, sendo o 
sentimento que mais se mostrou presente. Outros sentimentos mencionados foram insegurança, agressão, afastamento, vergonha, preconceito, solidão, desprezo, isolamento, angústia, ansiedade e mágoa. É importante relembrar que tais sentimentos não apareceram isolados, mas associados uns aos outros.

$\mathrm{O}$ medo apresentado pelos pacientes, segundo suas referências se fazia presente pelo fato dos mesmos terem medo de transmitir a doença, principalmente no que diz respeito a(o) parceira(o). Com medo de transmitir a doença e se sentir culpado, afastava-se de todos, ou seja da família e da(o) parceira(o), levando-o ao isolamento e conseqüentemente à solidão.

No que concerne às reações percebidas pelos pacientes, podemos observar que, de um total de 50 pacientes, $29(58,0 \%)$ referiram ter percebido reações manifestadas por outras pessoas, em relação à sua doença e 21 pacientes, ou seja 42,0\%, não perceberam qualquer tipo de reação.

Dentre as reações percebidas, as mesmas foram categorizadas em positivas, negativas e neutras. Quanto às percepções, $4(8,0 \%)$ referiram ter percebido reações positivas como por exemplo situações onde não só a família, mas também os amigos se aproximaram dele na tentativa de ajudá-lo e dar-lhe mais atenção.

Do total de 50 pacientes, 24 (48,0\%) perceberam reações negativas, onde surgiram reações das mais variadas.

Dentre os 24 pacientes, $3(6,0 \%)$ referiram ter "perdido o interesse sexual" pelo(a) parceiro(a); $3(6,0 \%)$ manifestaram insegurança e medo de se relacionar sexualmente com a parceira, pelo fato da possibilidade de transmitir a doença; $1(2,0 \%)$ referiu ter tido medo de se relacionar com a esposa e morrer após a relação. Dois $(4,0 \%)$ mencionaram que as pessoas queriam separar todos os seus objetos e os marcavam; 15 (30,0\%) referiram que, tanto os familiares como os amigos se afastavam, "desaparecendo" de suas casas e discriminando-os. Um $(2,0 \%)$ paciente referiu "não ter percebido nada", enquadrando-se no tipo de reação neutra.

Em geral, podemos notar que as reações negativas predominam pois, quando se trata de uma doença estigmatizante e embargada de preconceitos como a tuberculose, observamos que ela se sobrepõe aos sentimentos e laços que envolvem a família, os amigos e os parceiros.

No que diz respeito ao afastamento dos parceiros, podemos perceber que a "inibição do desejo sexual achase quase sempre associada à evitação do contato, que pode atingir a intensidade de uma evitação fóbica" (KAPLAN, 1983, p.105).

O medo e conseqüente afastamento pode ser uni e/ou bilateral e de acordo com observações feitas, a partir das respostas obtidas, nota-se que o primeiro origina-se, inicialmente e com freqüência, no(a) parceiro(a) e não no portador de tuberculose pulmonar.

Tal reação gera uma insegurança muito grande no casal, limitando a sua relação, chegando ao ponto de se evitarem física e sexualmente, pois segundo HOGAN (1980) na sexualidade existe uma mutualidade, onde se tem como centro o próprio eu e o outro e que um intercurso sexual não envolve apenas um encontro físico íntimo, mas também um encontro emocional.

As reações negativas interferem fortemente nas relações, provocando interferências e/ou modificações que geram o afastamento do casal, que se priva de se relacionar sexualmente, colocando assim, a tuberculose pulmonar como uma barreira entre os seus sentimentos e desejos.

Dos 50 pacientes questionados quanto ao conhecimento da família, a respeito da tuberculose pulmonar, $40(80 \%)$ referiram ter contado à família e aos amigos sobre a doença e 10 pacientes, ou seja, 20\% não notificaram a família a respeito da mesma. Dentre os 40 pacientes que notificaram a família, $20(50 \%)$ perceberam reações e 20 (50\%) não perceberam reações.

Dentre as reações percebidas pelos pacientes, encontramos as mais variadas situações, que envolvem direta ou indiretamente o medo das outras pessoas de adquirirem a doença.

Percebemos que os pacientes que comunicaram à família que haviam adquirido a doença, experienciaram preconceito ou rejeição. Preconceito pelo fato de a tuberculose pulmonar ser uma doença carregada de estigmas negativos e também pelo fato de apresentarem medo de se contaminar, culminando com a rejeição.

Concordamos com HERZLICH (1991), quando o autor denota que "o agir das pessoas é orientado pela compreensão que possuem do objeto que motivou a ação" (p. 18).

A tuberculose, quando surge inesperadamente, torna-se um forte objeto ou causa, modificando comportamentos e desviando as condutas pessoais, fazendo com que o indivíduo se afaste da razão, devido ao próprio medo, que mascara as atitudes no enfrentamento de uma doença transmissível.

Estudando o comportamento e atitudes de uma população envolvida com a tuberculose pulmonar, MATA \& JOSE (1985) evidenciou que a mesma possui uma imagem negativa da doença, o que resulta em rejeição social dos tuberculosos.

Percebemos que a tuberculose pulmonar desde o seu surgimento vem amedrontando a humanidade e talvez por ser uma doença tão antiga, o medo e a rejeição dos que convivem com ela nunca deixaram ou deixarão de existir, refletindo diretamente na vida do portador, forçando-o a experienciar afastamento e conseqüente isolamento social e/ou sexual. 
Em se tratando da caracterização do exercício da sexualidade, tentou-se reunir aspectos sobre a vida sexual dos portadores de tuberculose pulmonar, considerando-se os dois períodos.

Em se tratando dos tipos de parceria, nos 2 períodos, agrupando-se os mesmos, percebemos que antes da manifestação da tuberculose pulmonar haviam 8 $(16,0 \%)$ pacientes que possuíam parceria ocasional e 42 $(84,0 \%)$ parceira fixa, sendo ela unicamente a esposa, amasiada ou namorada. Após a manifestação da doença, notamos que $39(78,0 \%)$ mantiveram a sua parceira fixa, $4(8,0 \%)$ passaram a ter parceria ocasional e $7(14,0 \%)$ tornaram-se inativos sexualmente. Vale ressaltar que o fato de terem parceria, não significa necessariamente que os mesmos mantinham relações sexuais. Portanto consideramos como inativos, os indivíduos que não exerciam nenhuma prática sexual, em parceria ou por si só.

Convém notar que houve um aumento do número de pacientes que se tornaram inativos sexualmente, após a manifestação da doença e tais mudanças dos seus hábitos sexuais, podem estar diretamente ligadas ao aparecimento da tuberculose pulmonar.

No que concerne à freqüência das relações sexuais dos pacientes, os mesmos foram questionados quanto ao número de relações sexuais que mantinham com seu/sua parceiro(a), onde a parceria poderia ser fixa ou não.

Apresentamos na Tabela 1, as modificações ocorridas na freqüência das relações sexuais por dia, semana ou mês, após a manifestação da tuberculose pulmonar.

Tabela 1 - Distribuição das freqüências diária, semanal e mensal das relações sexuais dos portadores de tuberculose pulmonar, segundo os dois períodos em comparação. Ribeirão Preto, 1997

\begin{tabular}{lcccccccc}
\hline \multicolumn{1}{c}{ FREQÜÊNCIA DAS } & \multicolumn{2}{c}{ DIÁRIA } & \multicolumn{2}{c}{ SEMANAL } & \multicolumn{2}{c}{ MENSAL } & \multicolumn{2}{c}{ TOTAL } \\
\multicolumn{1}{c}{ RELAÇES } & $\mathbf{n}$ & $\mathbf{0}$ & $\mathbf{n}$ & $\mathbf{0}$ & $\mathbf{n}$ & $\mathbf{0}$ & $\mathbf{n}$ & $\mathbf{\%}$ \\
\hline INALTERADA & 8 & 16,0 & 10 & 20,0 & 2 & 4,0 & 20 & 40,0 \\
DIMINUIU & 5 & 10,0 & 5 & 10,0 & 1 & 2,0 & 11 & 22,0 \\
AUMENT OU & 0 & 0 & 3 & 6,0 & 0 & 0 & 3 & 6,0 \\
TORNARAM-SE & & & & & & & & \\
INATIVOS & 0 & 0 & 11 & 22,0 & 5 & 10,0 & 16 & 32,0 \\
\hline TOTAL & $\mathbf{1 3}$ & $\mathbf{2 6 , 0}$ & $\mathbf{2 9}$ & $\mathbf{5 8 , 0}$ & $\mathbf{8}$ & $\mathbf{1 6 , 0}$ & $\mathbf{5 0}$ & $\mathbf{1 0 0 , 0}$ \\
\hline
\end{tabular}

Independente do fato de as freqüências das relações sexuais serem diárias, mensais ou semanais, podemos observar, de um modo geral, que de acordo com o número total de pacientes, $20(40,0 \%)$ dos entrevistados não referiram qualquer alteração na freqüência das relações; $11(22,0 \%)$ referiram apresentar uma diminuição na freqüência das mesmas; $3(6,0 \%)$ revelaram ter aumentado a freqüência das relações, enquanto 16 $(32,0 \%)$ afirmaram não ter mais relações sexuais com a parceira após a manifestação da doença.

Este dado nos mostra que a tuberculose pulmonar interferiu, neste grupo de indivíduos, no interesse pelo(a) parceiro(a), refletindo assim nas relações que os indivíduos mantêm.

É importante ressaltar que tal desinteresse e conseqüente diminuição das relações sexuais não se dá somente pelo fato de o portador de tuberculose pulmonar apresentar-se debilitado; o que se mostra mais forte é o medo de transmitir ou de adquirir a doença.

$\mathrm{O}$ medo pode ser um sentimento bilateral ou recíproco, culminando com o afastamento do casal, refletindo conseqüentemente, no relacionamento e causando alterações na vida sexual de ambos.

Quando falamos em alterações na vida sexual, não tratamos somente da freqüência com que ocorrem as relações sexuais entre casais, mas também da mudanças provocadas em algumas das 3 fases da resposta sexual humana.

Baseadas no modelo trifásico da resposta sexual humana, o qual compreende as fases do desejo, da excitação e do orgasmo, observamos as mudanças provocadas em cada uma dessas fases, atendo-nos à presença ou ausência de determinada fase, pois tal modelo dá sentido e organiza os dados referentes à resposta sexual (KAPLAN, 1983). Concernente às mudanças relacionadas à $1^{a}$ fase ou seja, à presença ou ausência do desejo, obtivemos que 39 (78,0\%) pacientes referiram apresentar desejo, mesmo após a manifestação da doença; $6(12,0 \%)$ mencionaram a ausência do desejo, após a doença; 3 (6,0\%) apresentaram ausência de desejo antes de adquirir a doença e a presença do mesmo após a manifestação da mesma; $2(4,0 \%)$ restantes referiram apresentar ausência de desejo nos dois períodos.

No que diz respeito à freqüência da excitação nos dois períodos, encontramos que $38(76,0 \%)$ pacientes apresentaram-na antes e após a manifestação da doença; $9(18,0 \%)$ referiram não apresentar excitação no $2^{\circ}$ período, ou seja apresentavam excitação antes da 
manifestação da doença e após o aparecimento da mesma não mais a sentiam; $1(2,0 \%)$ mencionou não apresentar excitação no primeiro período e sim após o seu aparecimento; em 2 (4,0\%) pacientes, a excitação apresentou-se ausente em ambos os períodos.

Em relação à $3^{\circ}$ fase da resposta sexual humana, ou seja da fase do orgasmo, encontramos que $10(20,0 \%)$ referiram que antes de adquirir a doença obtinham orgasmo e que após o aparecimento da mesma o orgasmo tornou-se ausente; $1(2,0 \%)$ referiu obter orgasmo somente após a manifestação da doença e $2(4,0 \%)$ mencionaram a ausência do orgasmo nos dois períodos.

De acordo com os valores obtidos nas 3 fases, notamos que parte predominante dos pacientes conservaram o desejo, a excitação e o orgasmo, mesmo tendo adquirido uma doença transmissível, com caraterísticas de crônico degenerativa e sendo submetidos ao tratamento específico para a tuberculose pulmonar.

Três $(6,0 \%)$ indivíduos referiram ter apresentado o aparecimento do desejo; $1(2,0 \%)$ referiu o aparecimento da excitação e $1(2,0 \%)$ o aparecimento do orgasmo, após a manifestação da mesma.

Tal acontecimento pode estar relacionado ao fato de a isoniazida provocar, em alguns indivíduos, uma ligeira euforia, o que pode conseqüentemente, levar a uma alteração ou mesmo um aumento da libido, levando-o, assim, a apresentar um maior interesse sexual pelo(a) parceiro(a).

\section{CONSIDERAÇÕES FINAIS}

É interessante notar que quase todos os pacientes referiram conhecer a tuberculose pulmonar por este próprio nome enquanto outros, uma pequena porcentagem, referiram não conhecê-la por outro nome. Mas será que estes últimos não a chamam pelo próprio nome por realmente não saberem ou pelo fato de a doença ser embargada de estigma e preconceitos? Isto os forçaria a um ajustamento social pois talvez, se mencionassem o nome da doença a algum familiar ou amigo, seriam discriminados porque o estigma da doença e o preconceito com relação ao indivíduo doente ainda hoje persistem em nossa sociedade (BARREIRA, 1992).

Ressaltamos que os pacientes entrevistados encontravam-se em tratamento e/ou seguimento há mais de 3 meses nas referidas unidades, onde a mínima expectativa que se poderia ter é que eles já estivessem bem orientados quanto à doença.

Será que as orientações aos pacientes está sendo feita de forma adequada, verificando-se a efetiva compreensão dos mesmos?

O conhecimento empírico obtido por eles provinha de comunicações informais, sendo que os mesmos não demonstravam interesse em adquirir conhecimentos.

Notamos que as experiências vivenciadas pelos portadores estão diretamente ligadas ao convívio com os familiares e à parceria, surgindo assim sentimentos negativos.

A negatividade do sentimento pode ser também devido à associação que as pessoas fazem: "Se ele tem tuberculose, tem AIDS!"

A maior parte deles não revelou o diagnóstico à família e principalmente aos amigos, justamente pelo fato de terem medo de ser discriminados.

Vemos portanto, que as experiências vivenciadas pelos portadores de tuberculose pulmonar, estão muito mais relacionadas ao preconceito que a doença carrega consigo do que com a própria manifestação da doença.

A tuberculose pulmonar em si não provocou mudanças no tipo de parceria sexual mantida pelos pacientes, já que a maioria possuía uma parceria fixa e assim a manteve.

Há uma expressiva reflexão da doença na freqüência das relações sexuais, que diminuíram relativamente com o aparecimento da mesma (BERTAZONE, 1998). A manifestação da tuberculose pulmonar reflete no número de relações sexuais não somente pelo fato da mesma provocar uma debilidade no organismo, mas, a principal causa aqui em questão, é a diminuição da freqüência das relações sexuais, as quais neste contexto, estão submetidas a um preconceito existente. O preconceito gera o medo, que por sua vez, provoca alterações nos hábitos sexuais destes pacientes, refletindo no desejo, na excitação e no orgasmo.

Dados referentes a estas 3 fases nos mostraram que as suas freqüências se mantiveram, na maior parte inalterados, onde houve uma pequena mudança, ou seja, o desejo, a excitação e o orgasmo tornaram-se ausentes em uma pequena porcentagem dos pacientes. A ausência do orgasmo, depois do aparecimento da doença, tornouse mais evidente. Tal fato talvez esteja relacionado à debilidade do organismo provocada pela tuberculose pulmonar.

Os resultados obtidos no grupo estudado sugerem que a tuberculose pulmonar provoca alterações na vida sexual dos indivíduos, não só por ser uma doença crônico degenerativa, que debilita o indivíduo, provocando uma diminuição da sua capacidade física, mas também pelo fato de o medo, o preconceito e a discriminação envolverem a doença. Tais sentimentos não são provenientes somente do próprio paciente, mas também das pessoas que convivem com ele, inclusive de sua parceria, quando ela tem conhecimento a respeito da doença.

O que pode acontecer é que a sociedade provoque mudanças no comportamento de um indivíduo, sobretudo 
quando o mesmo adquire uma doença como a tuberculose pulmonar.

Os portadores de tuberculose pulmonar e seus familiares deveriam receber mais atenção no que diz respeito às orientações sobre a sua doença e principalmente um acompanhamento psicológico pois, a grande maioria não dispõe de conhecimento a respeito da relação da doença com a sua vida sexual.

A adequação destas orientações contribuiriam para uma socialização adequada dos mesmos, refletindo positivamente nos seus comportamentos sexuais, modificando suas atitudes e seus sentimentos negativos, provocando assim, uma mudança nos seus modos de ver e enfrentar a tuberculose pulmonar e a discriminação por ela provocada.

É premente a necessidade dos profissionais de saúde, onde destacamos o enfermeiro, envidar esforços no sentido de oferecer assistência sistematizada aos portadores de tuberculose pulmonar. A situação epidemiológica da doença é crítica e requer atenção especial dos profissionais de saúde, desde a formação acadêmica.
Enquanto doença cuja infecção pode ser contraída via ocupacional, exige dos profissionais de saúde conhecimento, habilidade e atitudes que visem ao emprego correto e humanístico das precauções e medidas para o estabelecimento do diagnóstico e tratamento precoce e monitorizado.

Os pacientes necessitam ser compreendidos e têm direitos, sobretudo a uma assistência e a um relacionamento interpessoal humanístico, isentos de preconceitos, rejeições e omissões.

Portanto, um dos passos fundamentais para a implementação de ações específicas ao portador de doenças transmissíveis é o conhecimento da realidade, é o identificar dos fatores que intervêm no experienciar o desenvolvimento desta patologia.

Com este trabalho, tivemos a feliz oportunidade de estudar tais aspectos que, sem dúvida somam subsídios para que os profissionais de saúde compreendam a experiência dos portadores de tuberculose pulmonar e possam trabalhar aspectos de sua sexualidade, com o intuito de promover uma assistência à saúde integral e de qualidade.

\section{THE SEXUALITY OF PEOPLE WITH PULMONARY TUBERCULOSIS CARED AT HEALTH BASIC UNITS AT RIBEIRÃO PRETO-SP}

This descriptive study was carried out in order to identify and analysing the knowledge that the patients with pulmonary tuberculosis have about the disease and the experiences which they have passed through; to identify some aspects related to their sexuality's changing after acquiring the pulmonary tuberculosis and characterizing the living experiences toward their family, the friends'/relatives' reactions and the emerging feelings. Fifty sexually active patients were interviewed. The results showed that $15(30,0 \%)$ patients with pulmonary tuberculosis did not have any knowledge about the disease and 32 (64,0\%) did not know how to prevent it. Among the fifty patients interviewed, 29 (58,0\%) have realized negative reactions. The pulmonary tuberculosis do not interferes directly only in these sexual and social people's lives, but also in their partners social lives. This fact has been contributing in order to decrease their sexual relations often.

KEY WORDS: pulmonary tuberculosis, emotion, nursing

\section{ASPECTOS GENERALES DE LA SEXUALIDAD DE LOS PORTADORES DE TUBERCULOSIS PULMONAR ATENDIDOS EN UNIDADES BÁSICAS DE SALUD DE RIBEIRÃO PRETO-SP}

Este estudio tuvo como objetivos identificar y analizar el conocimiento que los portadores de tuberculosis pulmonar tienen sobre la enfermedad, las experiencias vividas por ellos, enfatizando los cambios ocurridos en el ejercicio de la sexualidad, las reacciones de los familiares/amigos y los sentimientos que sobresalen a partir de estas experiencias. Fueron entrevistados cincuenta pacientes, sexualmente activos, portadores de tuberculosis pulmonar. Los resultados evidenciaron que 15 (30,0\%) de los portadores de tuberculosis pulmonar no tienen conocimiento sobre la enfermedad y 32 (64,0\%) no saben como prevenirla. De los 50 entrevistados, 29 (58,0\%) percibieron reacciones negativas, en donde, tanto el miedo del propio portador en transmitir la infección/enfermedad, como el miedo de la compañera sexual en adquirir la infección/enfermedad, pueden causar alteraciones en su vida social, afectiva y/o sexual, llevando a la disminución de la frecuencia de relaciones sexuales.

TÉRMINOS CLAVES: sexualidad, tuberculosis pulmonar, emociones negativas, enfermería 


\section{REFERÊNCIAS BIBLIOGRÁFICAS}

\section{BARREIRA, I.A. A enfermeira Ananéri no país} do futuro: a aventura da luta contra a tuberculose. Rio de Janeiro, 1992. 335p. Tese (Doutorado) Escola de Enfermagem Anna Nery, Universidade Federal do Rio de Janeiro.

02. BERTAZONE, E.C. O exercício da sexualidade de portadores de tuberculose pulmonar atendidos em unidades básicas de saúde de Ribeirão Preto-SP. Ribeirão Preto, 1998. p. 11. Dissertação (Mestrado) - Escola de Enfermagem de Ribeirão Preto, Universidade de São Paulo.

03. BRASIL. SUS. Casos de agravos e doenças infecciosas e parasitárias. Informe Epidemiológico, v. 6, n. 1, p. 2, Jan./Mar. 1997.

04. HERZLICH, C. A problemática da representação social e sua utilidade no campo da doença. Phisis Rev. Saúde Coletiva, v. 1, n. 2, p. 23-24, 1991.
05. HIJJAR, M.A. Controle de tuberculose no Brasil - a estratégia do plano emergencial. Bol. Pneum. Sanit., v. 5, n. 1, p. 98-107, Jan/Jun. 1997.

06. HOGAN, R.M. Human sexuality: a nursing perspective. Connecticut: Aplleton-CenturyCrofts, $1980.747 \mathrm{p}$.

07. KAPLAN, H.S. O desejo sexual: novos conceitos e técnicas da terapia do sexo. Trad. de Aurea Weissenberg. Rio de Janeiro: Nova Fronteira, 1983. cap. 6, p. 105-133.

08. MATA, S.; JOSE, I. Integrating the client's perspective in planning a tuberculosis education and treatment program in Honduras Special issue. The client's perspective in primary health care. J. Med. Anthropol., v. 9, n. 1, p. 57-64, 1985.

09. SCHAFFNER, W. Prevenção e controle da infecção hospitalar. In: WYNGAARDEN, J.B.; SMITH, L.H.; BENNET, J.C. CECIL - Tratado de Medicina Interna. 19. ed. Rio de Janeiro: Guanabara-Koogan, 1996. v. 2, cap. 289, p. 16241629. 